\title{
An experimental and theoretical investigation of
}

\section{droplet evaporation on heated hydrophilic and}

\section{hydrophobic surfaces}

\author{
Mustafa A. Kadhim ${ }^{a, b}$, Nikil Kapur ${ }^{a *}$, Jonathan L. Summers ${ }^{a}$ \& Harvey Thompson ${ }^{a}$

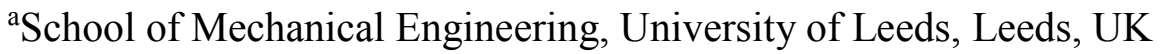 \\ ${ }^{\mathrm{b}}$ Mechanical Engineering Department, University of Babylon, Babylon, IRAQ \\ ${ }^{*}$ Corresponding Author (‥kapur@1eeds.ac.uk)
}

\begin{abstract}
The evaporation characteristics of sessile droplets on heated hydrophobic and hydrophilic surfaces are investigated. Results are reported for the evaporation of water droplet volumes covering a range of shapes dominated by surface tension or gravity and over a range of temperatures between $40^{\circ} \mathrm{C}$ and $60^{\circ} \mathrm{C}$. The weight evolution and total time of evaporation is measured using a novel self-contained heating stage on a high resolution analytical balance, which has advantages over visualization measurement techniques as it allows free choice of the initial droplet size and surface and the ability to record the droplet evaporation right through to the final stages of droplet life. Evaporation is modelled through a combination of a constant contact area and a constant contact angle model with the switch from the former to the latter occurring when the contact angle falls below its predetermined receding value. Theoretical results compare well with the experimental results for the hydrophobic substrate. However, a significant deviation is observed for the hydrophilic substrate due to the combined effects of droplet surface cooling due to evaporation and buoyancy effects which are not included in the model. The proposed method of using the stick-slip model offers a convenient means of modeling droplet evaporation by mimicking the drying modes based on initial measurements of the static and receding contact angles.
\end{abstract}




\section{KEYWORDS}

Droplet, evaporation, contact line, models, experiment

\section{INTRODUCTION}

The important role of droplet evaporation phenomena in many applications has motivated a wide range of experimental and theoretical investigations. These applications span industrial, medical and biological fields including DNA mapping ${ }^{1,2}$ biosensing $^{3}$, cooling ${ }^{4,5,6}$, cleaning $^{7}$, printing and painting $^{8}$. These studies explored the effect of the different parameters on the evaporation of droplets with particular focus on understanding the effect of substrate thermal conductivity, roughness and wettability on the evaporation process. However, droplet evaporation is still the focus of scientific investigation due to the associated complexity of interlinking flow dynamics, substrate physical surface chemistry and heat and mass transfer considerations.

A number of studies have explored the influence of the thermal conductivity of the substrate on the evaporation lifetime of droplets $9,10,11$. This has been found to be a key influence on the evaporation rate, for example David et al. ${ }^{9}$ and Sobac and Brutin ${ }^{11}$ have shown that there is a significant difference between the evaporation rate of non-metallic and metallic substrates with the same hydrophilic properties but no major differences between different metallic substrates with similar hydrophilic properties. Both experimental and numerical studies have examined the interaction between droplet evaporation and the wetting characteristics of the surfaces. The majority

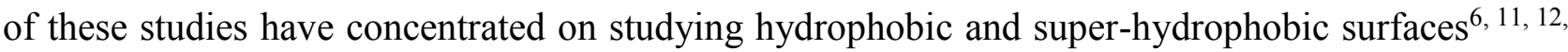
13, 14, 15 while comparatively few have investigated droplet evaporation over hydrophilic substrates 11,12. Studies concerning free droplet evaporation (no substrate temperature control) have received more attention $6,12,13,14,16,17,18,19,20$ than evaporation with controlled substrate temperatures ${ }^{11,15,16}$. The majority of studies have considered evaporation of droplets with volumes between $1 \mu l$ and $5 \mu l$ $6,11,13,14,15,19$, since these are consistent with the assumption of a spherical droplet cap, where 
surface tension dominates over gravity to yield a homogeneous and regular droplet shape. This then allows a visualization method to be adopted based on imaging the whole droplet profile from the side in order to determine the contact angle, contact radius, and droplet height $6,9,10,11,12,13,14,15,16$, 17, 18, 19, 20,21, 22, 23. The Young-Laplace model, valid for axisymmetric droplets, is the most common way of interpreting the data. However, this approach has limitations since estimates of the contact line position are very sensitive and are associated with large errors in the predicted contact line and contact angle ${ }^{24}$. Wilson et al. ${ }^{25}$ showed that the apparent contact angle (measured contact angle) depends on the resolution to which the angle is measured which is different than the actual value. Srinivasan et al. ${ }^{24}$ have shown that small variations in the lighting and camera focus can also lead to a difference of several degrees in the measured contact angle which in turn leads to significant errors in the calculated droplet volumes. The associated error increases at small contact angles and rates of droplet evaporation are often extrapolated from data where de-pinning occurs 9, 11, 26, 27 . There is an additional error associated with a non-circular contact line due to the surface roughness and surface impurities at small and large droplet sizes ${ }^{13,28,29,30}$.

Two main evaporation modes have been discovered by experimental observations which are related to the surface properties and characterised by the pinning and de-pinning properties of the droplet contact line: (i) constant contact radius mode (CCR), in which the contact angle of the droplet vanishes and the contact radius remains constant; (ii) the constant contact angle mode (CCA), in which the contact radius of the droplet vanishes and the contact angle remains constant ${ }^{31}$. However, another phase of evaporation has also been reported to occur in which both the contact angle and the contact radius collapse $\mathrm{e}^{20,32}$. The evaporation mode is largely controlled by the surface roughness ${ }^{33}$, wettability ${ }^{34}$ and surface energy ${ }^{35}$. Mollaret et al. ${ }^{10}$ found that for high surface energy, the pinning force increases with increased substrate temperature while there is little dependence on temperature for the low surface energy surfaces. Blake and DeConinck ${ }^{36}$ and Putnam et al. ${ }^{34}$ both examined the effect of surface wettability on the pinning and de-pinning forces and found that the 
pinning force dominates for contact angles lower than $90^{\circ}$ (hydrophilic surfaces) and reduces with increasing contact angle (hydrophobic surfaces). Therefore, hydrophobic surfaces are associated with the CCA evaporation mode 22,37 , although wettability is not the only defining factor for all the hydrophobic surfaces ${ }^{13}$. Kulinich and Farzaneh $^{38}$ determined that the mode of evaporation can be discovered from the contact angle hysteresis of the surface and cannot be explained by the initial contact angle of the droplet.

Characterizing surface properties and the interactions between the droplet and the substrate is necessary for numerical investigations. Bourges-Monnier and Shanahan ${ }^{39}$ derived an analytical expression for the evaporation of pinned droplets in the diffusion limited regime. This analytical expression was also successfully applied to describe the evaporation of the de-pinned droplets by Raj et al. ${ }^{40}$ and Gunjan et al. ${ }^{41}$. Similarly $\mathrm{Hu}$ and $\mathrm{Larson}^{42}$ proposed an approximate expression based on finite element analysis to describe a droplet evaporating in the pinned mode. Deegan et al. ${ }^{43}$ proposed a model to predict the local evaporation flux over the droplet surface as a function of the contact angle. Popov ${ }^{44}$ developed a model to calculate the total rate of evaporation based on the local evaporation flux model of Deegan et al. which was termed the vapour-diffusion model. Popov's model, which was originally developed to account for the deposition of solute resulting in a pinned contact line, underpins many studies for different surface wettabilities spanning hydrophilic $^{11,}{ }^{43}$, pinned hydrophobic ${ }^{11}$, de-pinned hydrophobic ${ }^{15,} 22$ and superhydrophobic $\operatorname{surfaces}^{13}$.

Note that the above models, which are based on the assumption of Fickian diffusion, are unable to model the flow of complex, multi-component droplet systems such as in water-glycol or waterethanol droplets ${ }^{45}$, fuel droplets ${ }^{46}$ or complex polymer systems ${ }^{47}$. Multi-component diffusion systems have been reviewed comprehensively by Bird \& Klingenberg ${ }^{48}$.

For the water droplet systems considered here, the vapour-diffusion model of Deegan et al. based on the assumption of Fickian diffusion, predicts the evaporation rate with reasonable accuracy for 
the hydrophobic ${ }^{15}$ and hydrophilic ${ }^{11,} 15$ surfaces but to significantly under predict the total evaporation time for super-hydrophobic surfaces ${ }^{15,22}$. Dash and Garimella ${ }^{22}$ used a scaling factor for the vapour-diffusion model to reduce the discrepancy of the predicted results for superhydrophobic surfaces. Gleason and Putnam ${ }^{49}$ introduced a modification for the local mass flux and the evaporation rate models by applying a temperature distribution across the liquid-vapour interface of the droplet. Pan et al. ${ }^{50}$ attributed the discrepancy of the vapour-diffusion model to evaporative cooling while Carle et al. ${ }^{51}$ claimed this under-estimation to be due to the natural convection driven by the buoyancy in the gas phase for the heated surfaces. However, others have attributed the under-estimation to the effect of substrate thermal conductivity which is not included in the underlying model ${ }^{11,13,26}$.

The mode of evaporation is vital in determining the evaporation mass flux and the total time of evaporation. Models focus either on a single mode of evaporation (CCR or CCA) that has the largest portion of the total evaporation time based on experimental visualisations ${ }^{11,15,22}$. Others have considered using a combination of models to describe the evaporation process where the droplet evaporates under the CCR mode and then shifts to the CCA mode based on experimental observations of the contact angle and the droplet radius to identify the point at which the mode of evaporation switches ${ }^{6,20,52,53}$. Some studies have developed an experimental control methodology (such as creating a trench in the substrate) to force the droplet to evaporate at constant contact radius allowing theoretical predictions for a specific model of operation ${ }^{49}$. Stauber et al. ${ }^{52}$ proposed a relationship between the initial contact angle and the point at which the droplet shifts into the depinning mode based on the experimental data of droplet evaporation from different studies in the literature. Recently, $\mathrm{Hu}$ and $\mathrm{Wu}^{53}$ examined theoretically the effect of different receding contact angles on the total time of evaporation assuming the droplet evaporates under pinned mode and then in the de-pinning mode once the receding contact angle was reached. It is also important to recognize that contact line shapes are also influenced by surface heterogeneity. A number of studies 
have shown that these can be tailored to specific shapes by carefully designing chemical heterogeneity on the surface ${ }^{29}$, by topographic patterning ${ }^{28}$, are highly susceptible to surface contaminants $^{41}$, and can be manipulated to flow in specific directions on asymmetric nanostructured surfaces ${ }^{30}$.

In the present study, the evaporation of sessile droplets over heated surfaces is examined experimentally using a self-contained heating stage allowing weight loss to be established until complete evaporation has occurred and theoretically using an evaporation model where the behaviour is switched based on a pre-measured receding contact angle. The originality of the present work is in its proposals for: (i) a new way of measuring the droplet evaporation using a precise measurement balance to overcome the aforementioned limitations of the conventional imaging methods; (ii) a new method of predicting the evaporation rates over the whole period of drying with a switch from CCR to CCA modes (stick-slip mode) based on the receding contact angle. Thus, whereas previously, the use of the stick-slip mode of evaporation have been reported by a few studies based on the experimental observation of the whole evaporation process, here we propose the use of a suction method of measuring the receding contact angle at the same time as measuring the initial contact angle to be fed in the model to define the point at which the mode of evaporation is shifted from CCR to CCA. This method will provide a much more convenient means of studying the evaporation of sessile droplets over any kind of surfaces and will only require simple initial measurements of the static and receding contact angles. Part of this paper is based on a previously published $\mathrm{PhD}$ thesis under the title "Holistic study of thermal management of direct liquid cooled data centres: from chip to environment ${ }^{54 \%}$.

This article is organized as follows: the first part includes description of the experimental and sample preparation methodologies for droplet evaporation. The second part discusses the theoretical analysis of the Deegan model for local evaporation flux, the Popov model of droplet evaporation and the stick-slip model implemented in this study. The third part presents the typical 
results of the Deegan model. The fourth part discusses the validation of the proposed model. Finally in the fifth part, the experimental and theoretical results of the droplet weight evolution are discussed for both surface tension-dominated and gravity-dominated droplets.

\section{EXPERIMENTAL METHODOLOGY FOR EVAPORATING DROPLETS}

The experiments deposit a controlled droplet volume on a heated substrate, which is then allowed to evaporate. The time evolution of the weight of the droplet is monitored and measured.

To investigate the influence of the sample substrate temperature, a compact self-contained unit was designed to carry the substrate sample and heat and control the temperature, as shown in Figure 1. The weight of the entire unit was such that it could be positioned on a stage of an accurate balance. The substrate is heated using a thick film electrical resistor powered by a small lithium polymer battery and controlled using an embedded microcontroller coded with a PID control loop (see Supporting Information). The circuit maintains a constant substrate temperature with a variation of $\pm 0.5^{\circ} \mathrm{C}$ by modulating the amount of current supplied to the resistor. Using the PID controller loop means that any temperature disturbance (e.g. reduced heat load during drying) will be automatically compensated for. The substrate under test is attached to the thermal resistor with a thin uniform layer of thermally conductive ethoxy paste (Electrolube TCER75S).

An analytical balance (METTLER TOLEDO XP 205) is used to measure and digitally record the droplet mass evolution as a function of time with a resolution of 10 micrograms. The design of the heated stage allows the self-contained unit to sit on the weighing pan, with sides around the balance to prevent disturbances by air currents.

A camera (Basler acA1300-30 $\mu \mathrm{m}$ and lens CCS SE-16SM) is used to obtain side and top views of the droplet. The recorded frames allow monitoring the droplet lifetime as well as the shape of the droplet especially at the final stages of droplet life. The droplet is created using a micropipette to control the droplet size, which is gently laid down on the plate sample to evaporate into the air inside a cubic cell of dimensions $\left(15 \times 18 \times 21 \mathrm{~cm}^{3}\right)$ to avoid any perturbation from potential 
external flow. To ensure repeatability of the results, each experiment is repeated four times. Before each measurement, a cleaning procedure is adopted by rinsing the substrate with deionized water and drying in an air stream.

The temperature and relative humidity inside the laboratory containing the balance was maintained at $21 \pm 0.2^{\circ} \mathrm{C}$ and $30 \pm 1 \%$, respectively. The effect of substrate temperature was investigated over five different temperatures: $40,45,50,55$ and $60^{\circ} \mathrm{C}\left( \pm 0.5^{\circ} \mathrm{C}\right)$. Deionized water droplets of four different initial volumes $(2,4,8$ and $30 \mu l)$ are investigated covering shapes dominated by surface tension through to those dominated by gravity; the Bond number $\left(B_{o}\right)^{55}$ varies from $B_{o}<1$ to $B_{o}>1$, respectively. The Bond number is defined by $B_{o}=\frac{\Delta \rho g R^{2}}{\gamma}$, where $\Delta \rho$ is the density difference between the water and air, $g$ is the gravitational acceleration, $\gamma$ is the surface tension between water and air at the substrate temperature, and $R$ is the droplet contact radius. 


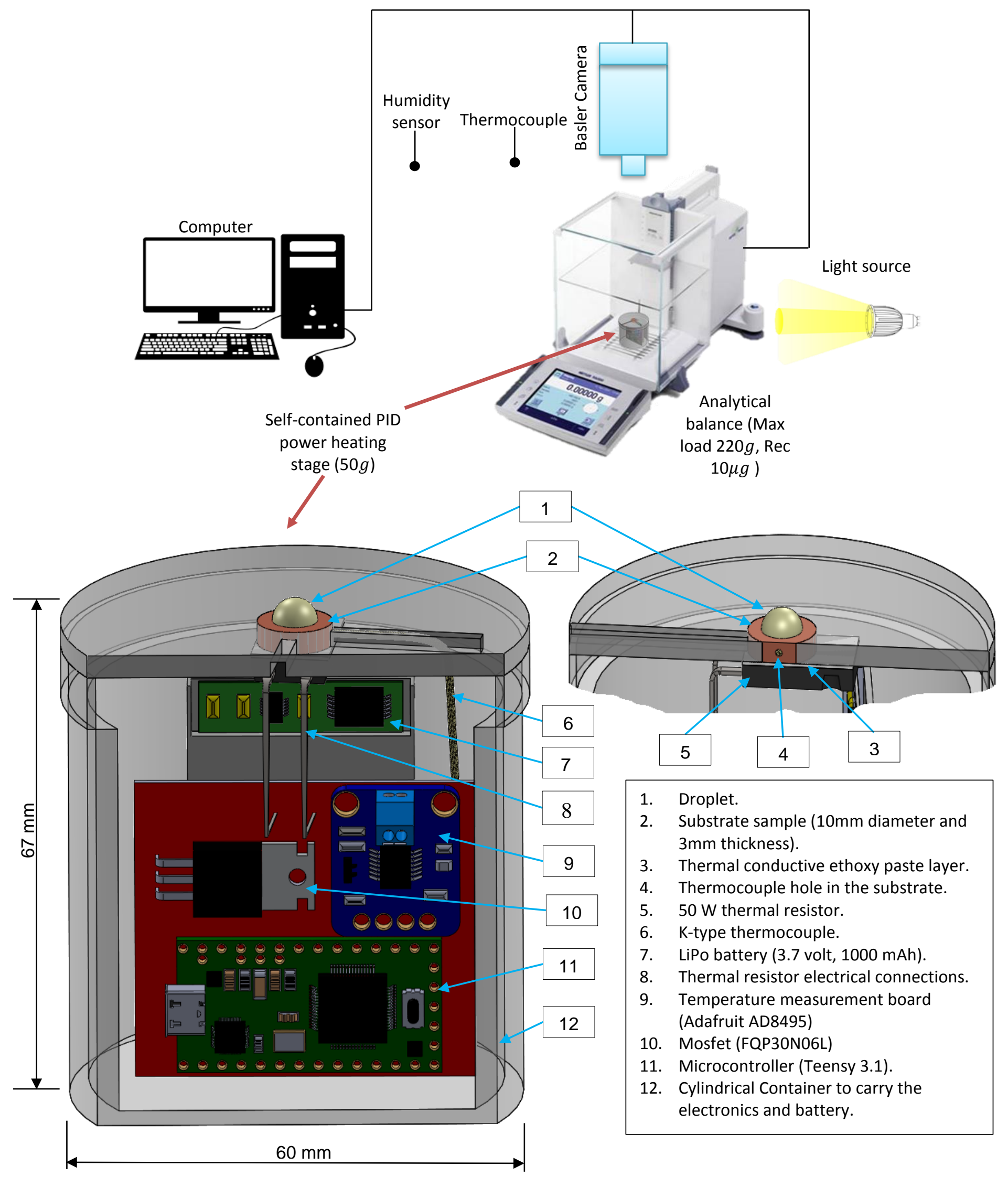

Figure 1 Experimental rig setup. 


\section{SAMPLE PREPARATION AND CHARACTERIZATION}

Two different substrates were studied, hydrophobic treated aluminium and hydrophilic treated aluminium. Disks of material were formed by machining aluminum bar of $10 \mathrm{~mm}$ diameter into disks of $3 \mathrm{~mm}$ thickness. The hydrophobic coated disks were polished from both sides with polishing paper of 1200 grade before being coated using Oxford nanoSystems ${ }^{56}$ nanoFLUX $^{\circledR}$ process to produce a dendritic surface nanostructure. Each disk has two holes (drilled from the sides) for temperature measurement, one of these holes is used to embed the electronic circuit thermocouple sensor whereas the other one is used for temperature calibration purposes.

The contact angle for all the samples was measured using CAM 200 from KSV. Each angle is reported as an average of 20 measurements taken at four locations across the surface. The static contact angles of the hydrophobic and hydrophilic aluminum surfaces were $120^{\circ} \pm 1.83$ and $49^{\circ} \pm$ 1.13 , respectively.

The receding contact angle was determined using a First Ten Angstroms (FTA) 4000 Microdrop where the droplet volume is reduced using a needle to create contact line motion (see Supporting Information). The measured receding contact angle was found to be $10^{\circ} \pm 0.6^{\circ}$ for the hydrophobic surface. For the hydrophilic surface, the contact line started receding at a value of $6^{\circ} \pm 2^{\circ}$. The error is due to the difficulty in visualising the small angle and is consistent with that previously observed ${ }^{34,36}$.

The surface roughness of the samples was determined using white light interferometry (Bruker NpFlex) (see Supporting Information). The root mean square roughness (RMS) was found to be 2.468 and $0.677 \mu \mathrm{m}$ respectively for the hydrophobic and hydrophilic surfaces. 


\section{THEORETICAL ANALYSIS: DIFFUSION DRIVEN DROPLET EVAPORATION MODEL}

The theoretical analysis presented here predicts the evaporation of a sessile droplet due to forced evaporation through heating of the substrate, allowing comparison with experimental data.

In the diffusion driven evaporation model, the limiting rate of mass transfer between the liquid and vapour medias is due to diffusion at the liquid-vapour interface. Fick's law of diffusion governs the evaporation of the droplet

$\frac{\partial c}{\partial t}=D \nabla^{2} c$

where, $D, c$ and $t$ are the diffusion coefficient, vapour concentration and time respectively.

The time scale analysis indicates that the droplet evaporation can be assumed to be quasi-steady when the droplet lifetime is much larger than the mass transfer Fourier number indicated by $(D /$ $R_{i}{ }^{2}$ ), where $R_{i}$ is the initial droplet radius. In this case, the transient term in equation (1) can be neglected, resulting in the Laplace equation

$\nabla^{2} c=0$.

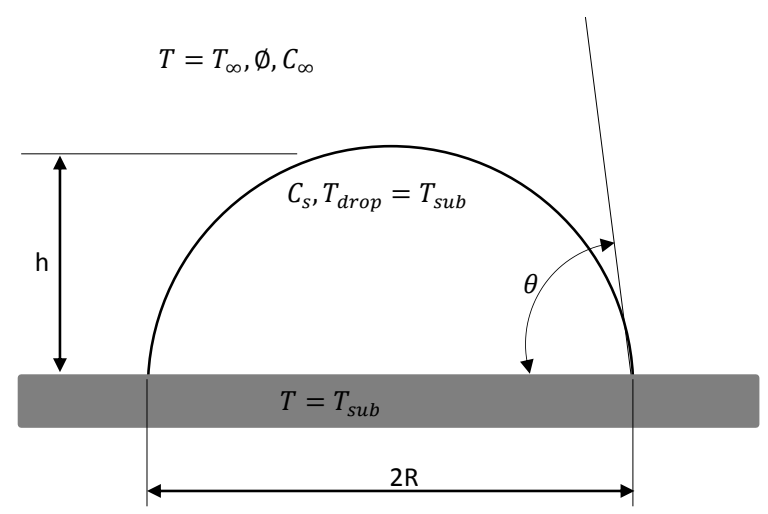

Figure 2 Droplet schematic and the boundary conditions

The droplet size adapted in this theoretical analysis is considered to satisfy the spherical cap assumption and to indicate symmetry across the central axis of the droplet. The boundary conditions (as shown in Figure 2) to satisfy the Laplace equation are (i) saturated vapour at the 
surface of the droplet at the substrate temperature $\left(c_{S}\right)$, (ii) the vapour concentration far from the droplet surface is defined by the ambient conditions $\left(c_{\infty}=\emptyset c_{\infty, S}\right)$, where $\emptyset$ is the relative humidity and $c_{\infty, S}$ is the saturated vapour concentration at the ambient temperature, (iii) all the phase change occurs across the liquid-vapour interface of the droplet, and (iv) the diffusion coefficient is calculated based on the substrate temperature. By employing a toroidal coordinate system as explained by Lebedev ${ }^{57}$, Laplace's equation can be solved for the vapour concentration around the droplet and the solution can be described as

$\frac{c(\alpha-\beta)-c_{\infty}}{c_{S}-c_{\infty}}=\sqrt{2 \cosh \alpha-2 \cos \beta} \times \int_{0}^{\infty} \frac{\cosh (\theta \tau) \cosh (2 \pi-\beta) \tau}{\cosh (\pi \tau) \cosh (\pi-\beta) \tau} P_{\frac{1}{2}+i \tau}(\cosh \alpha) d \tau$,

where $P_{\frac{1}{2}+i \tau}(\cosh \alpha)$ is the hyperbolic legendre function.

From equation (2) and (3), the Deegan model can be obtained which governs the local evaporation flux $^{43,44}$

$J(\alpha)=\frac{D\left(c_{s}-c_{\infty}\right)}{R}\left[\frac{1}{2} \sin \theta+\sqrt{2}(\cosh \alpha+\cos \theta)^{3 / 2} \times \int_{0}^{\infty} \frac{\tau \cosh (\theta \tau)}{\cosh (\pi \tau)} \tanh [(\pi-\right.$

$\left.\theta) \tau] P_{i \tau-\frac{1}{2}}(\cosh \alpha) d \tau\right]$

The total mass loss from a droplet during evaporation can be obtained by integrating the Deegan model, equation (4), over the liquid-vapour interface which results in Popov’s model 22,44

$\frac{d M}{d t}=\rho_{L} \frac{d V}{d t}=-\pi R D\left(c_{s}-c_{\infty}\right) f(\theta)$, where

$f(\theta)=\frac{\sin \theta}{1+\cos \theta}+4 \int_{0}^{\infty} \frac{1+\cosh 2 \theta \tau}{\sin 2 \pi \tau} \tanh [(\pi-\theta) \tau] d \tau$,

where $M, \rho_{L}, V$, and $R$ are the mass, density, volume, and contact radius of the droplet respectively.

The rate of mass change can be calculated as a function of base radius and contact angle. Two stages of evaporation are observed experimentally in which the major part of evaporation occurs with a constant droplet base radius, $\mathrm{R}$, and the contact angle a function of time, $\theta=\theta(t)$, until it reaches the receding contact angle, $\theta=\theta_{r}$, where the droplet starts de-pinning and the evaporation 
becomes a function of base radius only, $R=R(t)$ with the contact angle remaining fixed at the receding angle until evaporation is completed. The evolution of the contact angle during the first stage of evaporation of a pinned droplet and the second stage of evaporation of a de-pinned droplet can be respectively represented as

$\frac{d \theta}{d t}=\frac{D\left(c_{S}-c_{\infty}\right)}{\rho R_{i}^{2}}(1+\cos \theta)^{2} f(\theta)$

$\frac{d R}{d t}=\frac{D\left(c_{s}-c_{\infty}\right)}{\rho R^{2}} \frac{\left(1+\cos \theta_{r}\right)^{2}}{2+\cos \theta_{r}}\left[\frac{1}{1+\cos \theta_{r}}+4 \int_{0}^{\infty} \frac{1+\cosh 2 \theta_{r} \tau}{\sin \theta_{r} \sinh (2 \pi \tau)} \tanh \left[\left(\pi-\theta_{r}\right) \tau\right] d \tau\right]$.

The new mass of the droplet after each time step based on the new contact angle or the new base radius is calculated using the spherical cap assumption as $\left(M=\rho_{L} V\right)$

$M=\frac{\pi \rho R^{3}}{3} \frac{(1-\cos \theta)^{2}(2+\cos \theta)}{\sin ^{3} \theta}$.

The transient contact angle in equation (7) and the transient base radius in equation (8) were solved using the trapezoidal numerical integration method implemented in Matlab ${ }^{58}$. The numerical calculation procedures and the Matlab code are described in detail in the Supporting Information. 
INFLUENCE OF THE SURFACE WETTABILITY AND SUBSTRATE TEMPERATURE ON THE EVAPORATION FLUX.

The spatial variation of vapour concentration based on the Deegan model, equation (4), is shown in Figures 3 and 4 for the hydrophobic and hydrophilic surfaces, respectively. The arc length is calculated from the droplet surface radial distance (horizontal distance from the center of the droplet and the point at the droplet surface), which is described by $\frac{r}{R}=\sinh \alpha /(\cosh \alpha+\cos \theta)$, and the vertical coordinate, which is described by $\frac{z}{R}=\sin \theta /(\cosh \alpha+\cos \theta)$. The local evaporation flux is presented for all the cases as a function of the normalized arc length which is defined as the line around the gas-liquid interface from the point of the gas-liquid-solid contact line to the point of maximum height. This is normalized by the total arc length of the droplet. Substrate temperatures of 40,50 and $60^{\circ} \mathrm{C}$ and initial droplet sizes of $2 \mu l$ and $4 \mu l$ are chosen to illustrate the difference in the variation of the evaporation flux on the droplet surface.

The evaporation flux at the start of the evaporation process, calculated using equation (4) for droplet sizes of $2 \mu l$ and $4 \mu l$ on the hydrophobic surface with different substrate temperatures is shown in Figure 3. It shows that the evaporation flux is higher for the smaller droplet size; in all cases the maximum flux is at the point of maximum height of the droplet, and largely constant before rapidly reducing close to the contact line. This agrees with previous theoretical results ${ }^{22}$, which show that the evaporation flux by natural diffusion (no substrate heating) of the sessile droplet with hydrophobic contact angle remains almost uniform along the upper half of the droplet surface. The behaviour with the hydrophilic surface is significantly different. Figure 4 shows equivalent data to that in Figure 3, but for the initial conditions of the droplet with contact angle of $49^{\circ}$. It can be seen that the diffusion flux rapidly increases as the contact line is approached. In both cases reducing the temperature causes a reduction in the total evaporative flux, with the distribution of local flux transport remaining similar. 


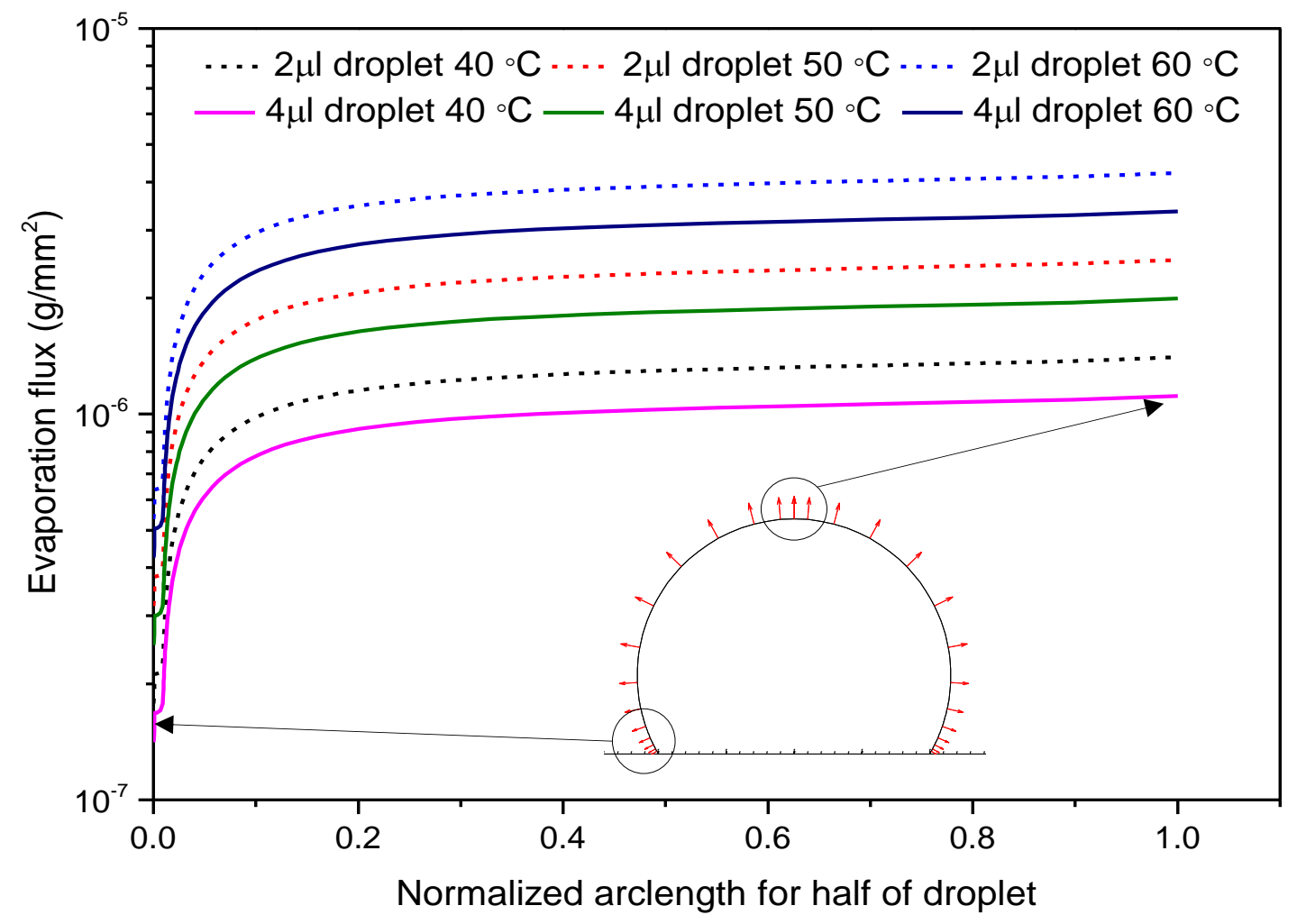

Figure 3 Initial local evaporation flux along the surface for $2 \mu l$ and $4 \mu 1$ water droplets for the hydrophobic surface for various substrate temperatures at contact angle of $120^{\circ}$.

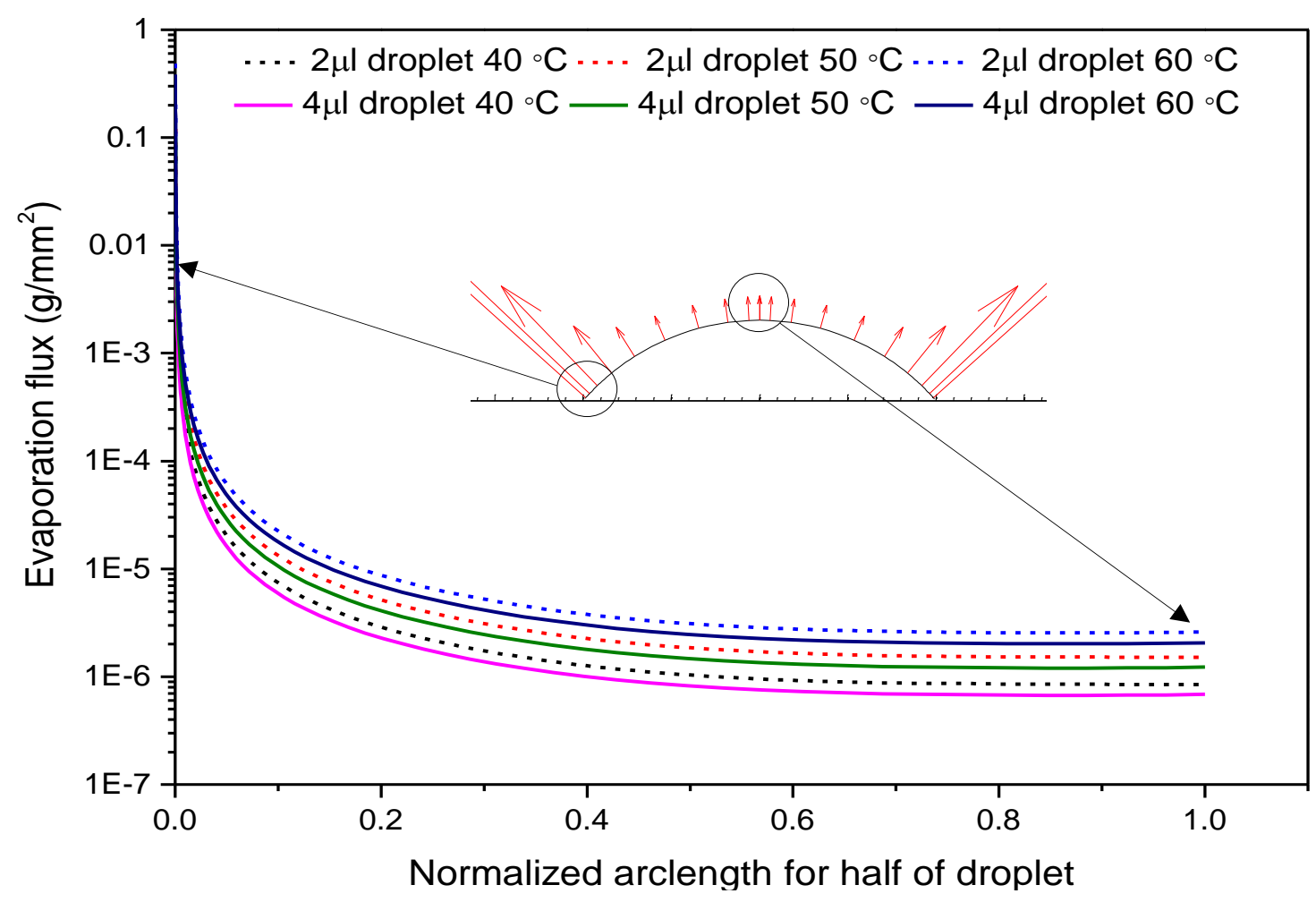

Figure 4 Initial local evaporation flux along the surface for $2 \mu 1$ and $4 \mu 1$ water droplets for the hydrophilic surface for various substrate temperatures at contact angle of $49^{\circ}$. 


\section{TOTAL TIME OF EVAPORATION}

The total time of droplet evaporation from a surface is highly dependent on the surface temperature and wettability. Figure 5 shows experimental measurements of the total time for the complete evaporation of 2, 4 and $8 \mu l$ water droplets, for the hydrophobic and hydrophilic surfaces, together with the predicted values using the diffusion only model. Increasing both the substrate temperature and wettability causes a reduction in the time taken for complete evaporation.

The stick-slip receding contact angle model (SSR) postulated here is used to predict evaporation, where the droplet initially evaporates under a constant contact radius mode until the contact angle falls below the receding angle, at which point the behaviour is switched to constant contact angle mode until complete evaporation. For most real surfaces, the modes of droplet evaporation are usually unknown a priori, however the combined model developed in this study (SSR) can capture the physical behaviour of droplet evaporation based on a pre-determined static and receding contact angle. This removes the requirement to visually monitor the droplet over its lifetime to establish the appropriate model to capture the mode of evaporation ${ }^{20,52,53}$.

In the case of the hydrophobic surface for the droplet sizes dominated by the surface tension force (2, 4 and $8 \mu l$ ), the total time of evaporation calculated by the vapour diffusion model based on the SSR mode is generally in excellent agreement with that measured experimentally, where an average relative deviation of less than $4 \%$ is observed as shown in Figures $5 \mathrm{a}-\mathrm{c}$. This suggests that the vapour diffusion model, based on the stick-slip behaviour where the slip happens when the receding contact angle is reached, succeeds in describing the entire influence of substrate heating throughout the evaporation process. In the case of the hydrophilic surface, the results of the model are presented for the range of measured receding contact angle between 4 and $8^{\circ}$. However, a considerable under prediction of $22 \%$ on average is observed when the surface is hydrophilic, as shown in Figures 5a-c. 
There are two possible explanations for these over-predictions. These are that: (i) the model does not include evaporative cooling ${ }^{15}$ over the height of the droplet resulting in an over-prediction of the surface temperature and consequently an under-prediction of the time of evaporation; (ii) buoyant convection ${ }^{59}$ resulting in an under-prediction of concentration gradient and an overprediction of the time of evaporation.

The hydrophobic droplets, presenting a higher droplet height to radius ratio than the equivalent (volume) hydrophilic droplet, creates conditions favourable for evaporative cooling, due to the distance between the hot base and cooler droplet surface - this may counter the buoyant convection thereby giving a closer match between model and experiment. The hydrophilic droplets will experience less evaporative cooling since the height to radius ratio is reduced; together with the higher surface temperatures driving buoyant convection.

Saenz et al.'s ${ }^{60} 3 \mathrm{D}$ simulations of evaporating, non-axisymmetric sessile droplets provides useful insight and context to the present results. Their Diffuse Interface simulations predicted the emergence of azimuthal currents and counter-rotating vortices within the bulk flow which could provide additional mechanisms for increasing heat transfer across the droplet. These would in turn lead to increased rates of evaporation which could potentially lower the predicted total evaporation times towards the experimental values obtained here. These effects are subtle and inter-related - but are supported by the stronger agreement between experimental and theoretical predictions for evaporation on hydrophobic surfaces when compared to hydrophilic surfaces, as shown in Figure 5. 


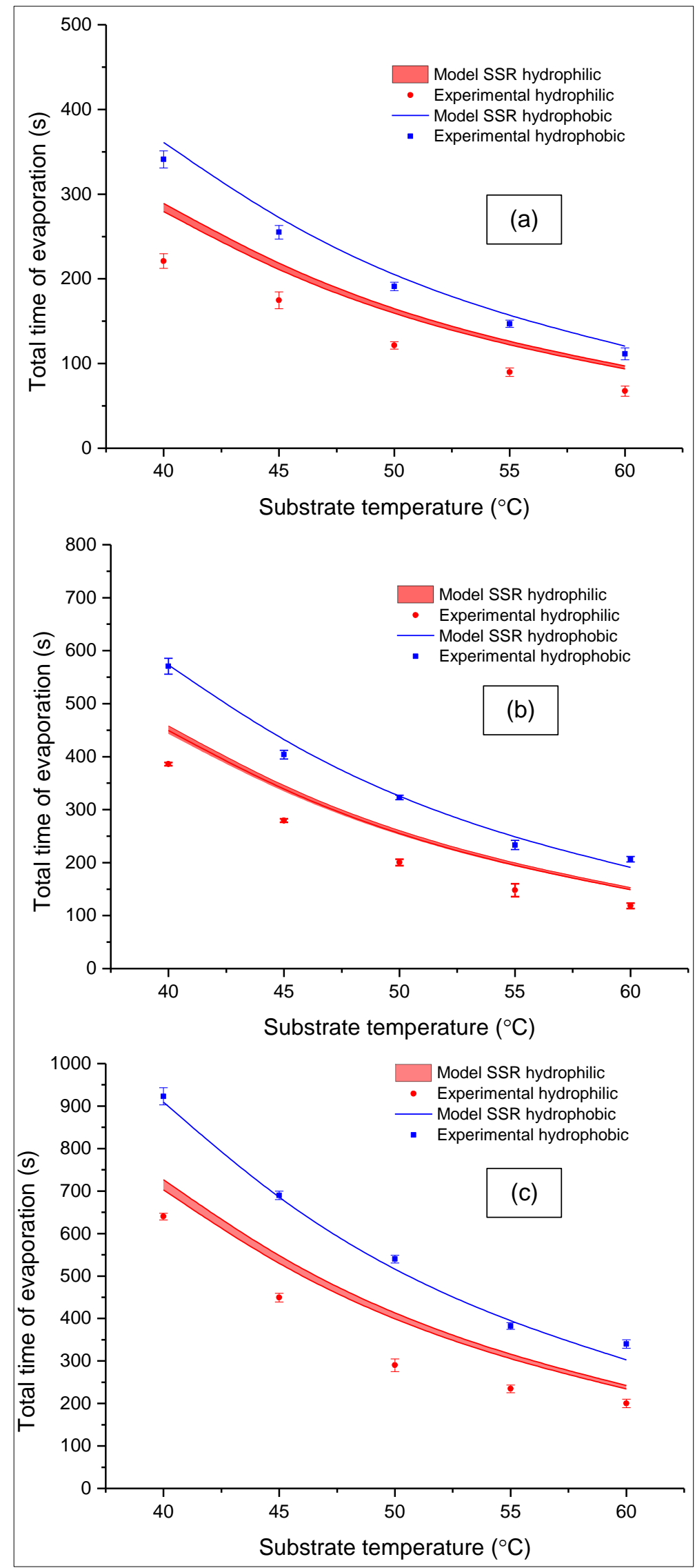

Figure 5 Total time for droplet evaporation on hydrophilic and hydrophobic surfaces as a function of substrate temperature. (a) $2 \mu 1$, (b) $4 \mu 1$ and (c) $8 \mu 1$. 


\section{EVAPORATION OF DROPLET SHAPES DOMINATED BY SURFACE TENSION}

Experimental and numerical results of the evaporation of water droplets are now presented for Bond numbers in the range between 0.07 and 0.7 allowing the droplet shape to be considered as a spherical cap.

The reduction in droplet weight with time during evaporation of the droplets over the hydrophobic surface for three different surface temperatures $\left(40,50\right.$ and $\left.60^{\circ} \mathrm{C}\right)$ are shown in figure 6 for droplet sizes of 2, 4 and $8 \mu 1$. The evolution of weight is consistent with earlier observations of droplet evaporation using the traditional visualization measurement technique over hydrophobic aluminium substrates coated with Teflon ${ }^{15,20}$ and PFC $^{11}$ coatings. The weight evolution of droplet evaporation over the hydrophobic surface was found to be predicted well by the vapour diffusion model based on the stick-slip behaviour mode (SSR). This is in agreement with earlier observations using the $\mathrm{CCA}^{15}$ and the $\mathrm{CCR}^{11}$ modes.

The weight evolution of the water droplet over time during evaporation over the hydrophilic surface for three different temperatures $\left(40,50\right.$ and $\left.60^{\circ} \mathrm{C}\right)$ is shown in Figure 7 for the droplet sizes of 2, 4 and $8 \mu l$. As the initial static contact angle is relatively small $\left(49^{\circ}\right)$, the surface area (both in contact with the surface and the air) is relatively large and the evaporation is much faster than that observed for an equivalent droplet on the hydrophobic surface. For the case of the hydrophilic surface (and as discussed previously), the vapour diffusion model based on the SSR mode underpredicts the experimental evaporation rate, herewith a percentage error of $22 \%$.

Visualisations of an $8 \mu l$ droplet, from the top and the side, on a heated substrate $\left(60^{\circ} \mathrm{C}\right)$ are shown in Table 1 for the initial droplet and at points from $90 \%$ to complete evaporation. This demonstrates that the droplet shape can be measured easily using the traditional visualization methods at the early stages, however, for the final $2 \%$ and $10 \%$ of the total time of evaporation of the hydrophobic and hydrophilic surfaces (respectively), there is a high associated error when calculating the droplet volume based on the contact angle or contact radius, as the droplet shape is very different from that 
of a spherical cap. This is consistent with the previous observations ${ }^{13,24}$. The high associated error in calculating the droplet size at the final stages of evaporation has resulted in a high uncertainty in calculating the total time of droplet evaporation and the rates of droplet evaporation are often extrapolated $^{6,13,27}$. The present method of weight measurement for the droplet evolution is shown to be superior regarding the independency on the droplet shape and the contact angle. This can also trace the evaporation process of any size of droplet which sits on any type of surface until it has evaporated completely, without the need for any approximation or extrapolation. 


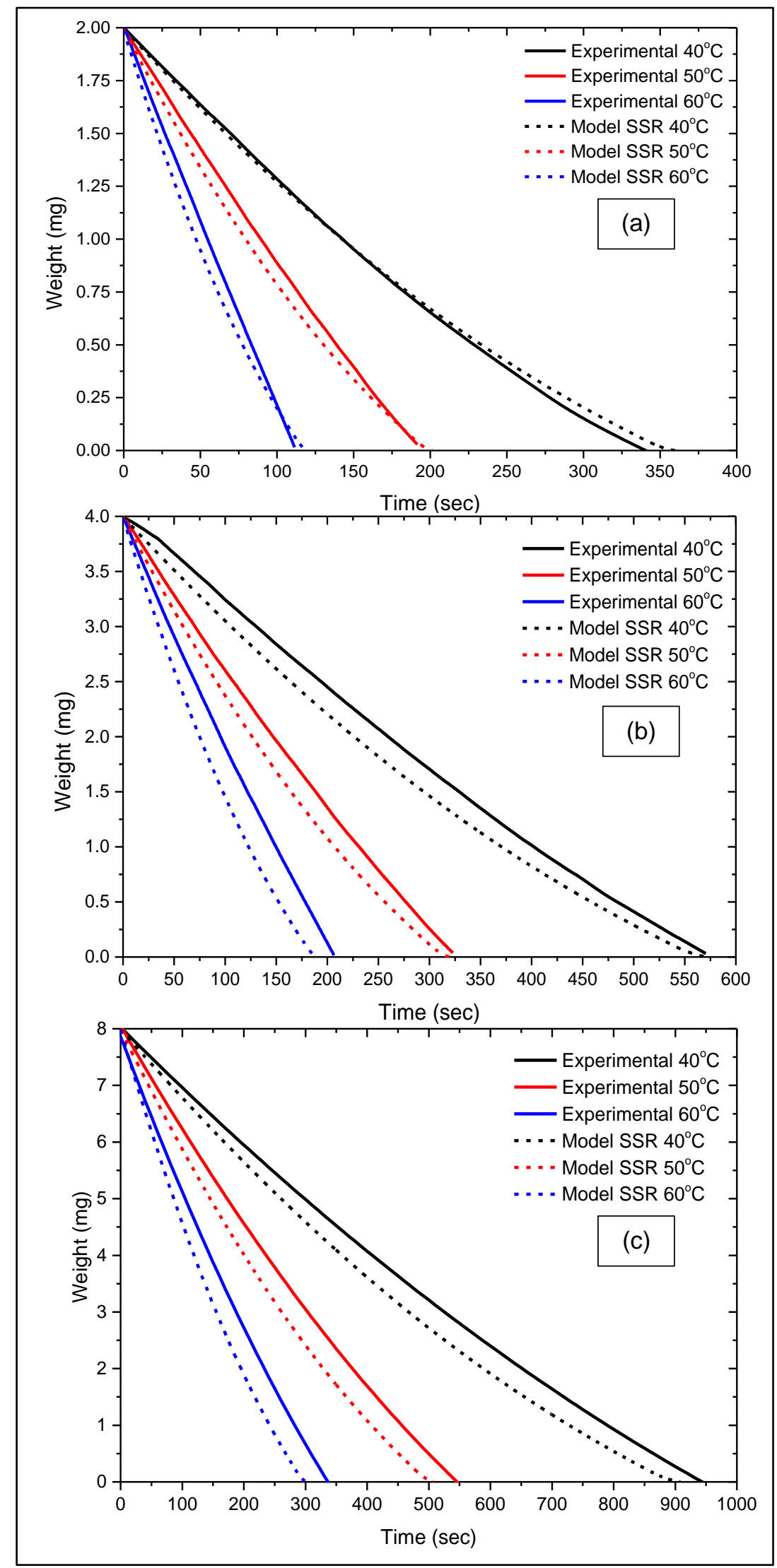

Figure 6 Measured and predicted (using stick-slip model) droplet weight evolution on the hydrophobic surface for a range of droplet sizes (a) $2 \mu 1$, (b) $4 \mu 1$ and (c) $8 \mu 1$. 


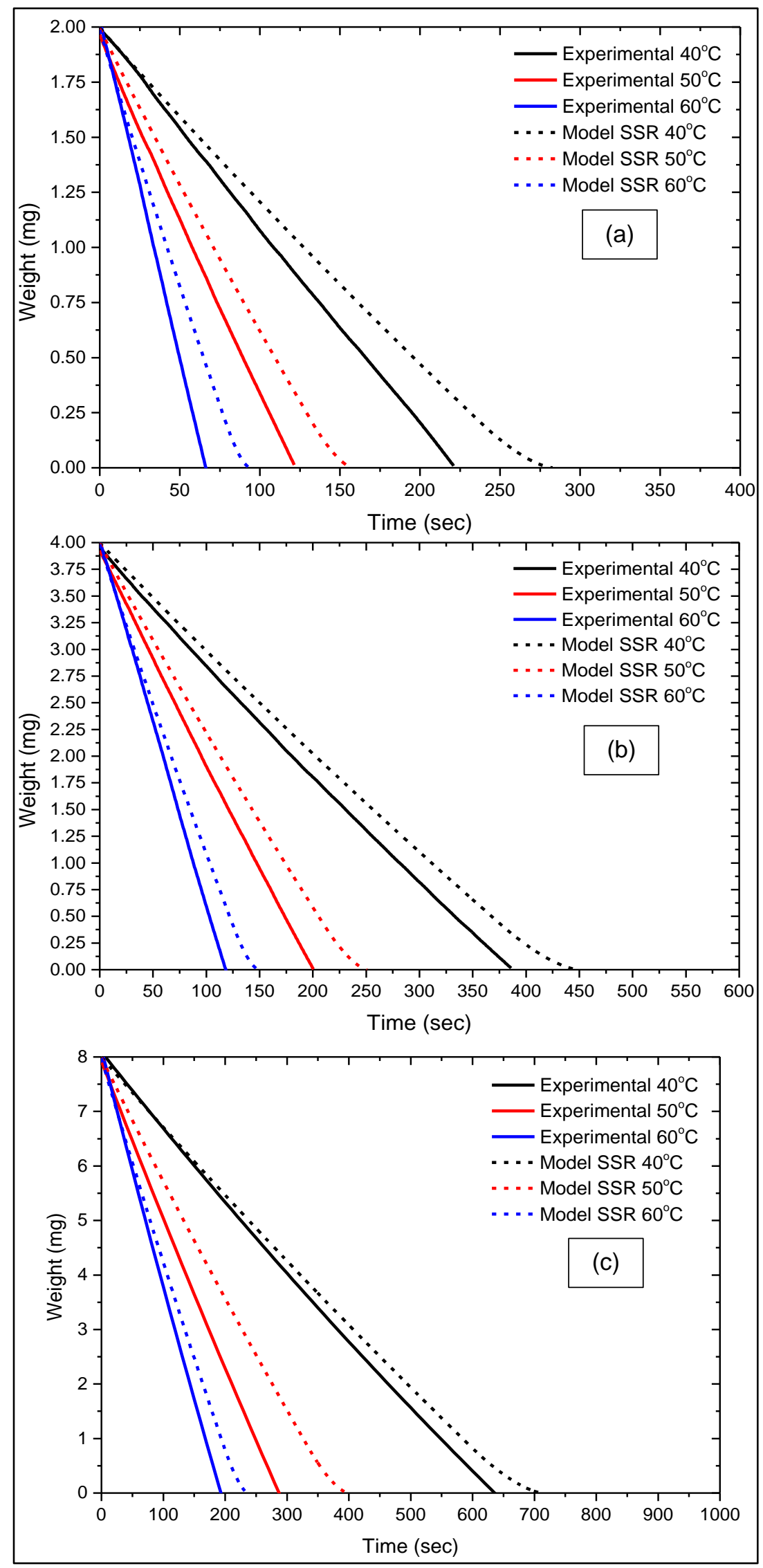

Figure 7 Measured and predicted (using stick-slip model) droplet weight evolution on the hydrophilic surface for a range of droplet sizes (a) $2 \mu 1$, (b) $4 \mu 1$ and (c) $8 \mu 1$. 
Table 1 Time-dependent images of an $8 \mu 1$ water droplet during the evaporation under the substrate temperature of $60^{\circ} \mathrm{C}$ for the hydrophobic and hydrophilic surfaces $\left(\mathrm{t}^{*}\right.$ is the normalized time which is the ratio of time from start of experiment to the total time of evaporation).

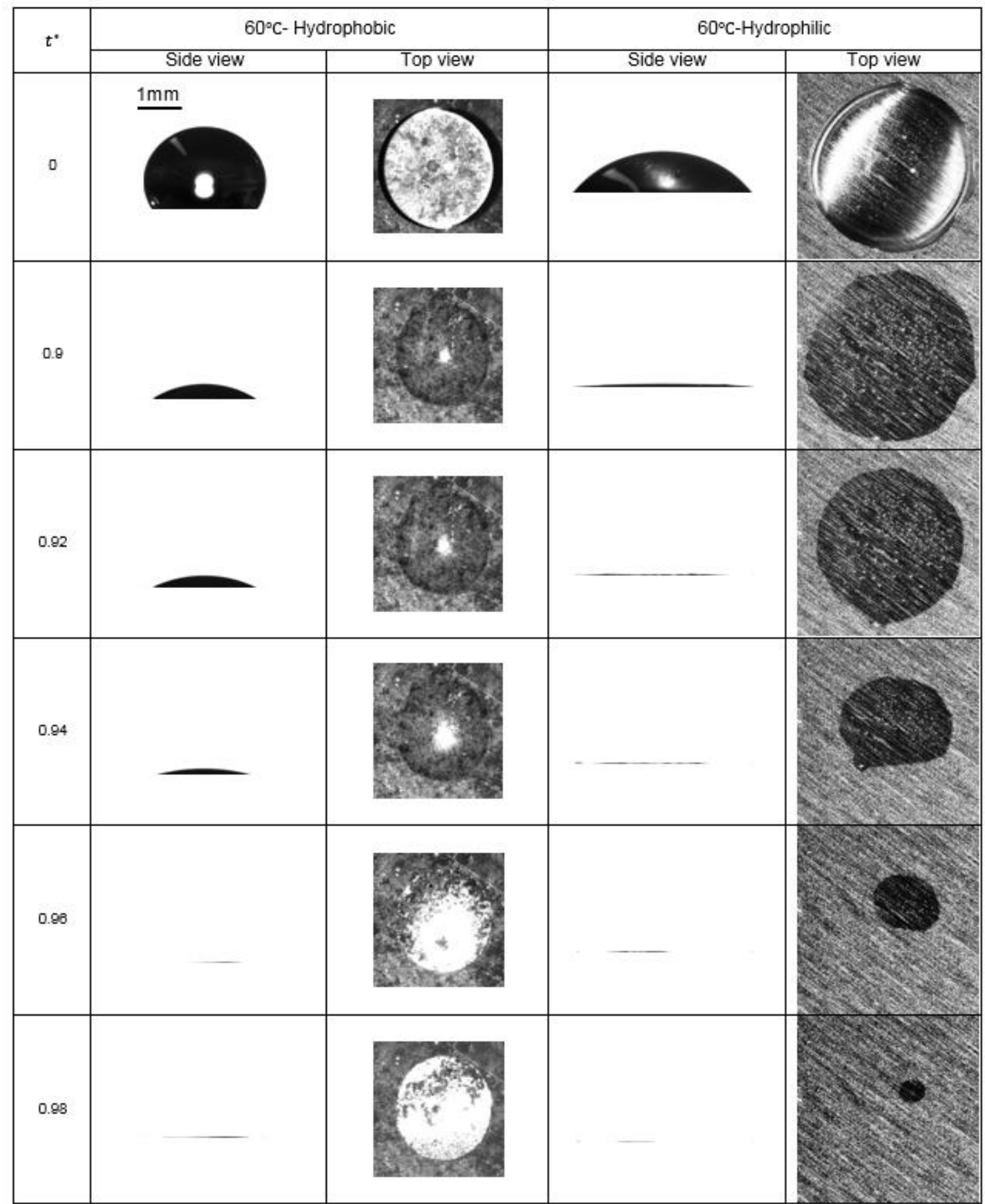




\section{EVAPORATION OF LARGE DROPLETS}

In this section, the evaporation of a relatively large water droplet volume $(30 \mu l)$ is studied. The mass evolution of the droplet with time is shown in Figures $8 \mathrm{a}$ and $8 \mathrm{~b}$ over the hydrophobic and hydrophilic surfaces respectively, for three different substrate temperatures $\left(40,50\right.$ and $\left.60^{\circ} \mathrm{C}\right)$.

The experimental results show that, for a given substrate temperature, the evaporation times on the hydrophobic surfaces are significantly greater than for the hydrophilic one with increases of around $75 \%, 60 \%$ and $40 \%$ for substrate temperatures of $40^{\circ} \mathrm{C}, 50^{\circ} \mathrm{C}$ and $60^{\circ} \mathrm{C}$ respectively. The chosen initial droplet volume leads to a higher ratio of gravity to surface tension forces which results in a Bond number of 0.78 for the hydrophobic surface and 1.7 for the hydrophilic surface. This is also associated with a larger contact radius compared to the capillary length $\left(k^{-1}\right)$ of the $\operatorname{droplet}\left(k^{-1}=\right.$

$\sqrt{\frac{\gamma}{\rho g}}$ ). However, for both the hydrophobic and hydrophilic surface, the proposed method of measurement based on the weight evolution successfully tracks the droplet mass for the studied range of temperatures.

The levels of agreement between theory and experiment for the two cases are, as expected, influenced by the Bond number since the model's spherical cap assumption is reasonable for the hydrophobic cases but is not appropriate for the hydrophilic ones. The SSR model was also used to predict the evaporation of the droplet as shown in Figure 8 and the average error in the hydrophobic case, shown in Figure 8a, is around 13\% but is much larger, $47 \%$, for the hydrophilic case as shown in Figure 8b. The high Bond number of 1.7 for the $30 \mu l$ droplet over the hydrophilic surface and its large contact radius compared to the capillary length results in a non spherical and flatter droplets which cannot be modelled accurately by the present approach. 


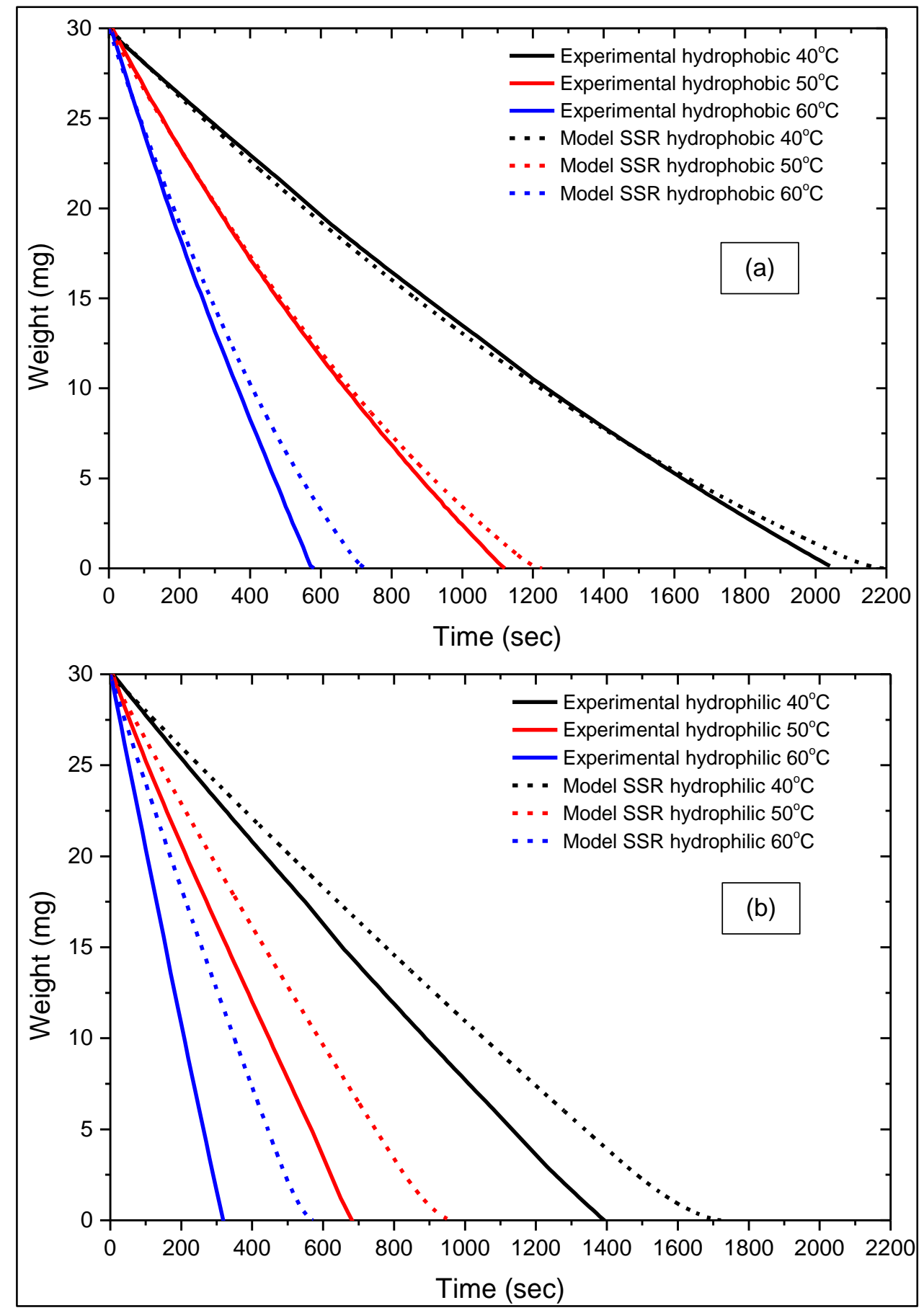

Figure 8 Measured and predicted (using stick-slip model) droplet weight evolution on (a) hydrophobic and (b) hydrophilic surfaces for a $30 \mu 1$ droplet. 


\section{CONCLUSION}

A comprehensive experimental and theoretical investigation of droplet evaporation on heated hydrophilic and hydrophobic surfaces is presented. Measurements of the droplet evaporation is achieved using a novel self-contained heating system, allowing characterization of the whole evaporation process for any initial droplet size. The experimental apparatus was successful in capturing actual evaporation rates without the need for imaging techniques.

A theoretical model is postulated here based on stick-slip behaviour (SSR) of the contact line. To fully identify the point of shifting from CCR mode to CCA mode in the model, the receding contact angle is measured using the needle suction method to be considered as the point where the slip mode (CCA) starts. The theoretical predictions agree well with the experimental results for the hydrophobic surface with an average percentage error of $4 \%$, however agreement is poorer for larger droplets for which the contact line is no longer circular and for hydrophilic surfaces. It would be instructive to assess the impact of the cross-droplet temperature variations, buoyancy effects within the air and non-circular contact lines using numerical techniques to define where regions of operability lie for simplified models, as has been done for free surface film flows ${ }^{48}$.

\section{ACKNOWLEDGMENT}

We would like to thank Dr Alexander Reip from Oxford nanoSystems Ltd. UK, for providing the hydrophobic coating, and Mr. Patrick Cotton from Airedale International Air Conditioning Ltd, UK for providing the coated samples. The authors would also like to thank Mr Kevin Gleason and Professor Shawn Putnam from the University of Central Florida, USA for the help with the MATLAB code to solve the Deegan model of local evaporation flux. NK thanks GSK and RAEng for funding his Research Chair. 


\section{SUPPORTING INFORMATION}

Detailed PID circuit along with C code. Detailed receding contact angle measurement procedure.

Scanning topography of the surfaces. Numerical calculation procedure for solving the Popov model along with the MATLAB script. This material is available free of charge in the Supporting Information file.

\section{REFERENCES}

1. Jing, J.; Reed, J.; Huang, J.; Hu, X.; Clarke, V.; Edington, J.; Housman, D.; Anantharaman, T. S.; Huff, E. J.; Mishra, B. Automated high resolution optical mapping using arrayed, fluid-fixed DNA molecules. Proceedings of the National Academy of Sciences 1998, 95 (14), 8046-8051.

2. Wu, A.; Yu, L.; Li, Z.; Yang, H.; Wang, E. Atomic force microscope investigation of large-circle DNA molecules. Analytical Biochemistry 2004, 325 (2), 293-300.

3. Ebrahimi, A.; Dak, P.; Salm, E.; Dash, S.; Garimella, S. V.; Bashir, R.; Alam, M. A. Nanotextured superhydrophobic electrodes enable detection of attomolar-scale DNA concentration within a droplet by non-faradaic impedance spectroscopy. Lab on a Chip 2013, 13 (21), 4248-4256.

4. Dhavaleswarapu, H. K.; Migliaccio, C. P.; Garimella, S. V.; Murthy, J. Y. Experimental investigation of evaporation from low-contact-angle sessile droplets. Langmuir 2009, 26 (2), 880-888.

5. Kumari, N.; Garimella, S. V. Characterization of the heat transfer accompanying electrowetting or gravity-induced droplet motion. International Journal of Heat and Mass Transfer 2011, 54 (17), 4037-4050.

6. Nguyen, T. A.; Nguyen, A. V.; Hampton, M. A.; Xu, Z. P.; Huang, L.; Rudolph, V. Theoretical and experimental analysis of droplet evaporation on solid surfaces. Chemical Engineering Science 2012, 69 (1), 522-529.

7. Blossey, R. Self-cleaning surfaces-virtual realities. Nature Materials 2003, 2 (5), 301-306.

8. Kim, D.; Jeong, S.; Park, B. K.; Moon, J. Direct writing of silver conductive patterns: Improvement of film morphology and conductance by controlling solvent compositions. Applied Physics Letters 2006, 89 (26), 264101.

9. David, S.; Sefiane, K.; Tadrist, L. Experimental investigation of the effect of thermal properties of the substrate in the wetting and evaporation of sessile drops. Colloids and Surfaces A: Physicochemical and Engineering Aspects 2007, 298 (1), 108-114.

10. Mollaret, R.; Sefiane, K.; Christy, J.; Veyret, D. Experimental and numerical investigation of the evaporation into air of a drop on a heated surface. Chemical Engineering Research and Design 2004, 82 (4), 471-480.

11. Sobac, B.; Brutin, D. Thermal effects of the substrate on water droplet evaporation. Physical Review E 2012, 86 (2), 021602.

12. Shin, D. H.; Lee, S. H.; Jung, J.-Y.; Yoo, J. Y. Evaporating characteristics of sessile droplet on hydrophobic and hydrophilic surfaces. Microelectronic Engineering 2009, 86 (4), 1350-1353.

13. Gelderblom, H.; Marin, A. G.; Nair, H.; van Houselt, A.; Lefferts, L.; Snoeijer, J. H.; Lohse, D. How water droplets evaporate on a superhydrophobic substrate. Physical Review E 2011, 83 (2), 026306.

14. Yu, Y.-S.; Wang, Z.; Zhao, Y.-P. Experimental and theoretical investigations of evaporation of sessile water droplet on hydrophobic surfaces. Journal of Colloid and Interface Science 2012, 365 (1), 254-259.

15. Dash, S.; Garimella, S. V. Droplet evaporation on heated hydrophobic and superhydrophobic surfaces. Physical Review E 2014, 89 (4), 042402. 
16. Sefiane, K.; Tadrist, L.; Douglas, M. Experimental study of evaporating water-ethanol mixture sessile drop: Influence of concentration. International Journal of Heat and Mass Transfer 2003, 46 (23), 4527-4534.

17. Sefiane, K.; Tadrist, L. Experimental investigation of the de-pinning phenomenon on rough surfaces of volatile drops. International Communications in Heat and Mass Transfer 2006, 33 (4), 482-490.

18. Lee, K.; Cheah, C.; Copleston, R.; Starov, V.; Sefiane, K. Spreading and evaporation of sessile droplets: Universal behaviour in the case of complete wetting. Colloids and Surfaces A: Physicochemical and Engineering Aspects 2008, 323 (1), 63-72.

19. Dunn, G.; Wilson, S.; Duffy, B.; David, S.; Sefiane, K. The strong influence of substrate conductivity on droplet evaporation. Journal of Fluid Mechanics 2009, 623, 329-351.

20. Stauber, J.; Wilson, S.; Duffy, B.; Sefiane, K. On the lifetimes of evaporating droplets. Journal of Fluid Mechanics 2014, 744, R2.

21. Sefiane, K.; Bennacer, R. Nanofluids droplets evaporation kinetics and wetting dynamics on rough heated substrates. Advances in Colloid and Interface Science 2009, 147, 263-271.

22. Dash, S.; Garimella, S. V. Droplet evaporation dynamics on a superhydrophobic surface with negligible hysteresis. Langmuir 2013, 29 (34), 10785-10795.

23. Bazargan, V. Effect of substrate cooling and droplet shape and composition on the droplet evaporation and the deposition of particles. Doctor of Philosophy, The University Of British Columbia2014.

24. Srinivasan, S.; McKinley, G. H.; Cohen, R. E. Assessing the accuracy of contact angle measurements for sessile drops on liquid-repellent surfaces. Langmuir 2011, 27 (22), 13582-13589.

25. Wilson, M. C.; Summers, J. L.; Shikhmurzaev, Y. D.; Clarke, A.; Blake, T. D. J. P. R. E. Nonlocal hydrodynamic influence on the dynamic contact angle: Slip models versus experiment. 2006, 73 (4), 041606.

26. Chen, Y.; Hu, W.; Wang, J.; Hong, F.; Cheng, P. Transient effects and mass convection in sessile droplet evaporation: The role of liquid and substrate thermophysical properties. International Journal of Heat and Mass Transfer 2017, 108, 2072-2087.

27. Lopes, M. C.; Bonaccurso, E.; Gambaryan-Roisman, T.; Stephan, P. Influence of the substrate thermal properties on sessile droplet evaporation: Effect of transient heat transport. Colloids and Surfaces A: Physicochemical and Engineering Aspects 2013, 432, 64-70.

28. Courbin, L.; Denieul, E.; Dressaire, E.; Roper, M.; Ajdari, A.; Stone, H. A. Imbibition by polygonal spreading on microdecorated surfaces. Nature materials 2007, 6 (9), 661.

29. Raj, R.; Adera, S.; Enright, R.; Wang, E. N. High-resolution liquid patterns via three-dimensional droplet shape control. Nature communications 2014, 5, 4975.

30. Chu, K.-H.; Xiao, R.; Wang, E. N. Uni-directional liquid spreading on asymmetric nanostructured surfaces. Nature materials 2010, 9 (5), 413.

31. Park, J. K.; Ryu, J.; Koo, B. C.; Lee, S.; Kang, K. H. How the change of contact angle occurs for an evaporating droplet: effect of impurity and attached water films. Soft Matter 2012, 8 (47), 11889-11896.

32. Nguyen, T. A.; Nguyen, A. V. Increased evaporation kinetics of sessile droplets by using nanoparticles. Langmuir 2012, 28 (49), 16725-16728.

33. Pittoni, P. G.; Chang, C.-C.; Yu, T.-S.; Lin, S.-Y. Evaporation of water drops on polymer surfaces: pinning, depinning and dynamics of the triple line. Colloids and Surfaces A: Physicochemical and Engineering Aspects 2013, 432, 89-98.

34. Putnam, S. A.; Briones, A. M.; Byrd, L. W.; Ervin, J. S.; Hanchak, M. S.; White, A.; Jones, J. G. Microdroplet evaporation on superheated surfaces. International Journal of Heat and Mass Transfer 2012, 55 (21-22), 5793-5807.

35. Bormashenko, E.; Musin, A.; Zinigrad, M. Evaporation of droplets on strongly and weakly pinning surfaces and dynamics of the triple line. Colloids and Surfaces A: Physicochemical and Engineering Aspects 2011, 385 (1-3), 235-240.

36. Blake, T.; De Coninck, J. The influence of solid-liquid interactions on dynamic wetting. Advances in colloid and interface science 2002, 96 (1-3), 21-36. 
37. Anantharaju, N.; Panchagnula, M.; Neti, S. Evaporating drops on patterned surfaces: Transition from pinned to moving triple line. Journal of colloid and interface science 2009, 337 (1), 176-182.

38. Kulinich, S.; Farzaneh, M. Effect of contact angle hysteresis on water droplet evaporation from super-hydrophobic surfaces. Applied Surface Science 2009, 255 (7), 4056-4060.

39. Bourges-Monnier, C.; Shanahan, M. Influence of evaporation on contact angle. Langmuir 1995, 11 (7), 2820-2829.

40. Raj, R.; Enright, R.; Zhu, Y.; Adera, S.; Wang, E. N. Unified model for contact angle hysteresis on heterogeneous and superhydrophobic surfaces. Langmuir 2012, 28 (45), 15777-15788.

41. Gunjan, M. R.; Raj, R. Dynamic Roughness Ratio-Based Framework for Modeling Mixed Mode of Droplet Evaporation. Langmuir 2017, 33 (28), 7191-7201.

42. Hu, H.; Larson, R. G. Evaporation of a sessile droplet on a substrate. The Journal of Physical Chemistry B 2002, 106 (6), 1334-1344.

43. Deegan, R. D.; Bakajin, O.; Dupont, T. F.; Huber, G.; Nagel, S. R.; Witten, T. A. Contact line deposits in an evaporating drop. Physical Review E 2000, 62 (1), 756.

44. Popov, Y. O. Evaporative deposition patterns: spatial dimensions of the deposit. Physical Review $E$ 2005, $71(3), 036313$.

45. Diddens, C.; Kuerten, J.; van der Geld, C.; Wijshoff, H. Modeling the evaporation of sessile multicomponent droplets. Journal of Colloid and Interface Science 2017, 487, 426-436.

46. Shiadeh, E.; Vahid, S. Development of multi-component evaporation models and 3D modeling of NOx-SCR reduction system. 2011.

47. Curtiss, C.; Bird, R. B. Multicomponent diffusion in polymeric liquids. Proceedings of the National Academy of Sciences 1996, 93 (15), 7440-7445.

48. Bird, R. B.; Klingenberg, D. J. Multicomponent diffusion-A brief review. Advances in water resources 2013, 62, 238-242.

49. Gleason, K.; Putnam, S. A. Microdroplet evaporation with a forced pinned contact line. Langmuir 2014, 30 (34), 10548-10555.

50. Pan, Z.; Dash, S.; Weibel, J. A.; Garimella, S. V. Assessment of water droplet evaporation mechanisms on hydrophobic and superhydrophobic substrates. Langmuir 2013, 29 (51), 15831-15841.

51. Carle, F.; Sobac, B.; Brutin, D. Experimental evidence of the atmospheric convective transport contribution to sessile droplet evaporation. Applied Physics Letters 2013, 102 (6), 061603.

52. Stauber, J. M.; Wilson, S. K.; Duffy, B. R.; Sefiane, K. On the lifetimes of evaporating droplets with related initial and receding contact angles. Physics of Fluids 2015, 27 (12), 122101.

53. Hu, D.; $\mathrm{Wu}, \mathrm{H}$. Volume evolution of small sessile droplets evaporating in stick-slip mode. Physical Review E 2016, 93 (4), 042805.

54. Kadhim, M. A. K. Holistic Study of Thermal Management in Direct Liquid Cooled Data Centres: from the Chip to the Environment. PhD, University of Leeds 2018, http://etheses.whiterose.ac.uk/21185/1/Holistic\%20study\%20of\%20thermal\%20management\%20in\%20dir ect\%20liquid\%20cooled\%20data\%20centres\%20from\%20chip\%20to\%20the\%20environment.pdf.

55. Berthier, J. Micro-drops and digital microfluidics; William Andrew2012.

56. Oxford nanoSystems. http://oxfordnanosystems.com/.

57. Lebedev, N. N. Special functions and their applications; Prentice-Hall, Inc: United States of America, 1965. p 322.

58. MATLAB. Reference manual. The Mathworks Inc. Natick, Massachusetts, United States 2013.

59. Girard, F.; Antoni, M.; Faure, S.; Steinchen, A. Influence of heating temperature and relative humidity in the evaporation of pinned droplets. Colloids and Surfaces A: Physicochemical and Engineering Aspects 2008, 323 (1), 36-49.

60. Sáenz, P.; Sefiane, K.; Kim, J.; Matar, O.; Valluri, P. Evaporation of sessile drops: a three-dimensional approach. Journal of Fluid Mechanics 2015, 772, 705-739. 

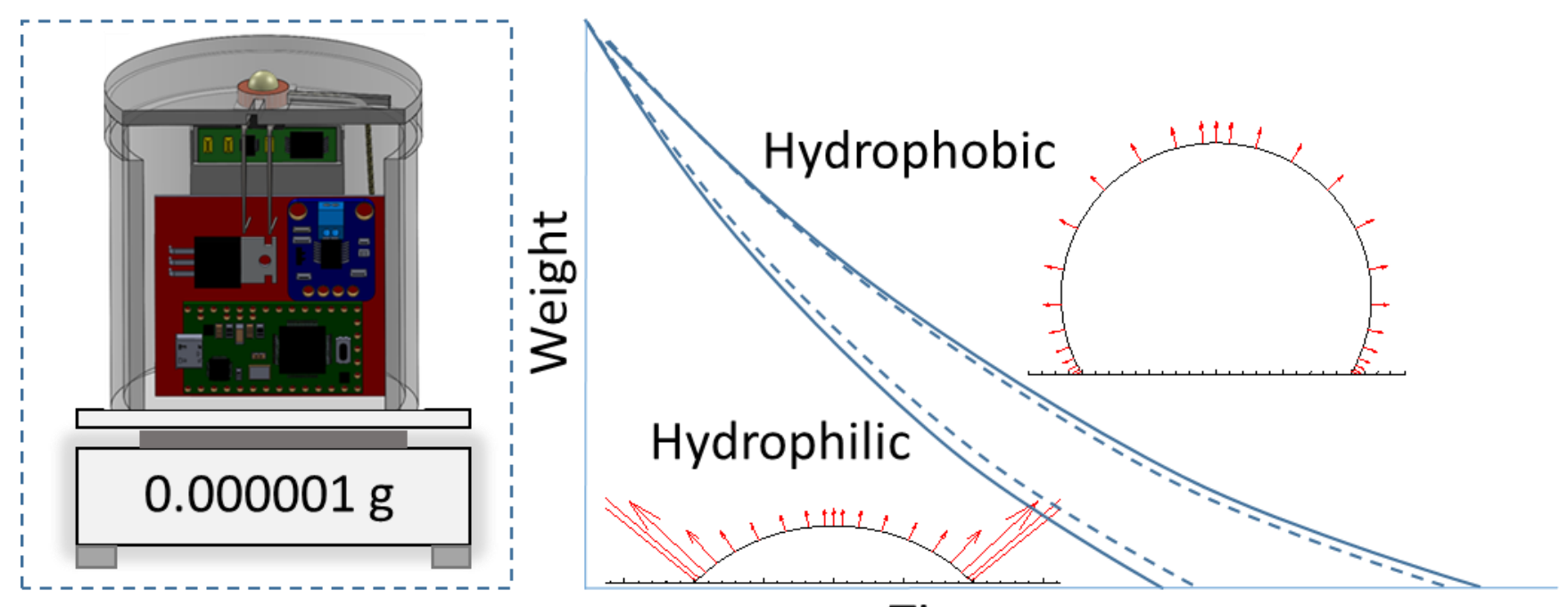

Time 